\title{
Glosse
}

\section{Günter Frankenberg Das Leben als Sitzblockade oder: Laepples Welt}

Wer sich erinnert, wird von der Ferne des Geschehenen eingeladen, dieses in ein mildes Licht zu tauchen. Mit der Vollendung von Vergangenheit will sich jene Regung des Gemüts einstellen, die wir Nachsicht nennen. Bei ein wenig philosophischer Bildung auch Toleranz. Diese Tugend schlägt keine alten Schlachten und lindert den Schmerz vergangener Niederlagen. Ja, sie erlaubt, die Gegner von einst großmütig zu umarmen. Über ihre Irrtümer und Fehler gnädig hinwegzusehen. Und demütig die eigene Position in Frage zu stellen. Solch verzeihendes und zugleich selbstkritisches Erinnern kann freilich nur gelingen, wenn das Geschehene dem Sich-Erinnernden zur persönlichen Genugtuung gereicht. Wenn der allfällige Kampf um Anerkennung von einigem Erfolg gekrönt war. Fehlt es dagegen an derartiger Genugtuung, dann sitzt der Stachel des Ressentiments tief im Gemüt und vergiftet Nachsicht wie Großmut. Stets sind es die Ungekrönten, die auf Rache sinnen. Der unstillbare Drang nach Anerkennung treibt die Verkannten im Modus der Erinnerung zur Abrechnung, und komme diese auch noch so spät. Endlich wollen sie ihre Leistungen gewürdigt wissen.

Eben so liest sich denn auch die »Erinnerung an das Recht«. Zu Papier gebracht und auf über zweihundert Druckseiten ausgebreitet vom ehemaligen Richter zu Schwäbisch Gmünd, Werner Offenloch. ${ }^{\mathrm{I}}$ Er kennt weder Verjährung noch Milde. Mit seiner literarischen Wiederaufnahme der Verfahren in Sachen Sitzblockaden gegen die Nachrüstung will er abstrafen. Die Verteidigung des Rechts, so wie er es sieht und sah und sprach, erlaubt keine Nachsicht.

Am Anfang war Laepple. Zunächst war es nur ein Fall: Der Bundesgerichtshof für Strafsachen hatte am 9. August $1969 \mathrm{zu}$ urteilen. Über den Studenten Laepple. Der hatte sich etwa drei Jahre zuvor mit Gesinnungsgenossen nach Art der damals angesagten Revolte auf den Straßenbahnschienen niedergelassen. So geschehen zu Köln. Zum Protest gegen die geplante Erhöhung der Tramtarife. Die Staatsanwaltschaft klagte an. Wegen Aufruhrs - damals noch strafbar. Und wegen Nötigung. Das Landgericht zu Köln vermochte Gewalt und folglich einen Aufruhr nicht zu erkennen. Ebenso, wenngleich anders begründet, kam es auch hinsichtlich der Nötigung zum Freispruch. Es ließ das Sich-Hinsetzen während der ersten fünfzehn Minuten als Demonstration durchgehen. Für die Zeit danach stellte das Landgericht eine Entschuldigung aus. Wegen eines unvermeidbaren Verbotsirrtums. Nicht so der Zweite Strafsenat des BGH. Er fand, für Strafrichter wie O., die richtigen Worte für das inkriminierte Verhalten. Aus dem Sich-Niederlassen wurde eine Blockade, aus passiver Resistenz eine Gewaltnötigung. Der BGH erkannte die tatbestandliche Gewalt darin, dass die Studenten »nur mit geringem körperlichen Kraftaufwand einen psychisch determinierten Prozeß in Lauf setzten «. ${ }^{2}$ Geboren war der vergeistigte Gewaltbegriff.

\footnotetext{
I Werner Offenloch, Erinnerung an das Recht. Der Streit um die Nachrüstung auf den Straßen und vor den Gerichten, Tübingen (Mohr Siebeck), 2005, 2 I6 S., 34.00€.

2 BGHSt 23, 46/54.
} 
Entgegen der Auffassung des Reichsgerichts im legendären Sargträger-Fall³ trat die psychische Zwangswirkung nunmehr gleichberechtigt neben »die Inbewegungsetzung des körperlichen (physischen), äußeren (mechanischen) Zwanges gegen Personen $« 4$ und teilte dessen indizielle Wirkung für die Verwerflichkeit der Nötigung. Recht so, meint Richter O.

Mit dieser Entscheidung erblickte das Laepple als Maßeinheit für die Berechnung eben dieser geistig-psychischen Zwangswirkung das Licht der Rechtswelt. Und gestattete, komplizierte Sachlagen more physico-metrico, also wissenschaftlich exakt, zu erfassen. Im Fall der Straßenbahnblockierer wird man im Hinblick auf den Fahrer der Tram rückwirkend I Laepple ansetzen dürfen, zumal die Kölner Ratsmitglieder, die eigentlichen Adressaten des Protests, fern vom Tatort weilten. Bei den Sitzblockaden in Mutlangen wären wegen des aufgeheizten Klimas eher 2 Laepple zu veranschlagen. Das dramatische Sich-Anketten an das Tor der Wiederaufbereitungsanlage von Wackersdorf sollte auf der nach oben offenen Laepple-Skala wohl mit 3 oder mehr Maßeinheiten bedacht werden. Laepple als Fall und Maß - das ist die Welt des Richters O.

In Mutlangen sollten Pershing-Raketen mit Atomsprengköpfen stationiert werden, wogegen sich alsbald Protest erhob. In der »Erinnerung an das Recht « fungiert die schwäbische Kleinstadt Mutlangen als Stichwort, Chiffre, Karma und Menetekel. Wer sich am Standort Mutlangen zum Protest niederließ, über den saß der gestrenge Richter O. im nahen Schwäbisch Gmünd alsbald zu Gericht. Hier hielt er die Stellung des Rechts. Vielleicht sollten wir sagen: Hier verteidigte er den Nötigungstatbestand des $\mathbb{} 240$ StGB im leicht verschärften Geist von Laepple gegen friedensbewegte Angreifer wie auch juristische Abweichler. Verschärft, weil Richter O. es vorzog, auf die Körper der Protestierenden statt auf die psychische Zwangswirkung abzustellen.

Die Klientel von O. betrieb den Widerspruch und Widerstand gegen die atomare Nachrüstung - jenes »Monstrum der westlichen Zivilisation« (Johan Galtung), das in Mutlangen, aber auch anderswo, sein fürchterliches Haupt erhob. Heinrich Böll, Walter Jens, Robert Jungk, Petra Kelly und viele Namenlose verschrieben sich dem Protest und übersahen, wie ihr Amtsrichter weitsichtig immer schon wusste, »den Systemkonflikt zwischen totalitärer und freier Welt«. »Bar jeden Verständnisses für die auf Frieden und Freiheit drängenden Politiker, « so O., orchestrierten sie zivilen Ungehorsam und »forensisches Pressuring «. Letzteres klingt, wenn man obszöne Assoziationen übergeht, nach einer Praktik im Umfeld von Wellness, meint freilich nur den auch medial vermittelten Druck auf die Gerichte.

Doch Richter O. hielt Stand. Er flüchtete nicht. Im Auge des Orkans verurteilte er furchtlos. Nach Maßgabe der - für ihn - seit Laepple gefestigten Rechtsprechung. »Wegen gemeinschaftlicher Nötigung zum Nachteil der betroffenen Fahrzeuglenker «, wie es nüchtern in den nicht ohne Verfasserstolz im Zentrum des Buches abgedruckten Entscheidungsgründen eines seiner Urteile aus dem Jahre 1990 heißt. Er entschied ganz »positivistisch«, Fall um Fall: Die sitzenden Täter übten Gewalt aus, indem sie »ein körperliches Hindernis bildeten « und ihre Nötigungsopfer so an der Weiterfahrt hinderten. Selbst sechs Minuten Wartezeit waren für den gestrengen Richter kein Pappenstiel. Selbst Fahrer einer Panzerkolonne, wie alle Opfer - vorsichtshalber? - nie befragt, seien in verwerf-

3 RGSt 45, I I/ I 56 (Zusammenrottung einer Menschenmenge, um die Sargträger daran zu hindern, einen mutmaßlichen Selbstmörder in geweihter Erde zu bestatten).

4 So das RGSt 45 , i 56. 
licher Weise genötigt worden. Also musste, weil für »ein gedeihliches Zusammenleben der Menschen unverzichtbar «, Strafe sein.

Gewiss, J'accuse ist nicht der Gestus des ehemaligen Richters O. Er hat es weniger mit Emile Zola als mit Kohlhaas. Denn dass er Recht hatte, möchte er denn doch nicht ungesagt lassen. Auf seiner Reise zum »Wesen des Rechts « macht er seiner Protestklientel, der kommentierenden Rechtswissenschaft und, in Umkehrung der instanziellen Hackordnung, seinen Obergerichten den Prozess. Nach einem Ausflug in die Welt der ihm genehmen Rechtsprinzipien listet er pedantisch die Verfehlungen seiner Gegner auf. Da bleibt keine Auge trocken.

Untergerichte, die in Sitzdemonstrationen keine Gewalt oder jedenfalls nichts Verwerfliches hatten erkennen können, 5 Recht-fertiger alle, müssen sich vorhalten lassen, dem Zeitgeist auf den Leim gegangen zu sein und dem »forensischen Pressuring « nachgegeben zu haben. Den sonst geschätzten BGH trifft der Vorwurf, in einem Blockade-Verfahren nach Renegatenart von seinem eigenen, dem Laepple-Kurs, ohne Not abgewichen zu sein. Hatten sich doch die Oberrichter »bequemt «, die Verwerflichkeit in $\ 240$ II StGB als, horribile dictu, unbestimmten Rechtsbegriff auszuzeichnen. ${ }^{6}$ Härter noch trifft der Bannstrahl des ehemaligen Richters allerdings das Bundesverfassungsgericht. Mehrmals mit Sitzdemonstrationen à la Mutlangen befasst und selten einer Meinung, ${ }^{7}$ wird es von dem erinnerungsseligen Amtsrichter nachgerade abgewatscht: Die verfassungsrichterlichen Sprüche verlören sich in »Umgereimtheiten, Doppelbödigkeiten und Widersprüchen«. Den Untergerichten - wie man hinzufügen möchte: an der Front - hätten sie »Steine statt Brot « gegeben und sie, insbesondere ihn, den Richter O., »im Regen stehen lassen«. Das OLG Stuttgart, als Revisionsinstanz schicksalhaft dem Amtsgericht Schwäbisch Gmünd übergeordnet, geißelt O., es sei in seiner Spruchpraxis den »Blockiererkreisen« allzu sehr entgegen gekommen. Wie das? Das OLG habe den Tatgerichten »eine fast endlose Liste von (zu prüfenden) Tatumständen vorgegeben«, damit Freisprüche in Kauf genommen und prinzipienlos die »Rechtslogik « verraten. ${ }^{8}$ So spricht ein Verkannter.

Wenig hält der Praktiker O. von den Theoretikern, so sie ihm widersprechen. Nicht einmal in der Rückschau will er die normative Komplexität von Sitzdemonstrationen sehen. Und weigert sich, altruistisch motivierte Nötiger von egoistischen, Nah- von Fernzielen zu unterscheiden. Ungereimt erscheint ihm, der sich auf Gewalt einen schlichten Reim gemacht hat, ziviler Ungehorsam. Für ihn nicht einmal ein Begriff, der Probleme anzeigt. Freudig zitiert O. dessen Verächter. Und genüsslich jene, die ihm mit einem »Recht « auf Ungehorsam in die Hände spielen. Trotz allem Prinzipienbeiwerk und Exkursen ins alte Rom, von wo er Ulpian etc. als Kronzeugen nach Schwäbisch Gmünd herbeizitiert, mag er vom Lagerdenken nicht lassen. Den Recht-Gläubigen, wie der in der letzten Sitzblockade-Entscheidung des Bundesverfassungsgerichts arg gebeutelten Richterin Haas, öffnet er die Tür zur Wärmestube der Verkannten. Einen nicht unerheblichen Teil der Dissidenten straft er, kritikscheu, mit Verachtung, indem er ihren Argumenten noch die Dignität einer Fußnote

\footnotetext{
ऽ Dokumentiert bei Günter Frankenberg, Passive Resistenz ist keine Nötigung. Untergerichte wider herrschende Rechtsprechung zu $\ 240 \mathrm{StGB}, \mathrm{KJ}$ i 985 , 30 I ff.

6 BGHSt 34, 7I (Vorlagebeschluss des OLG Köln).

7 BVerfGE 73, 206 (Großengstingen); 76, 2 I I (Bastian); Kammerbeschluss des Ersten Senats, BVerfG JR I 991, I 3 ff.; BVerfGE, 92, I (Großengstingen); I04, 92 (Wackersdorf).

8 Insbesondere: OLG Stuttgart, Beschl. v. I 8.9.1987 (3 Ss 369/87); Urt. v. 21.7.1988 (3 Ss 3 I I/88); Urt. v. 28.2.1989 (3 Ss 345/88); und besonders »unverblümt« OLG Stuttgart, Beschl. v. I 2.5.1 993 (3 Ss 475/90).
} 
verweigert. ${ }^{9}$ Oder zeiht sie, soweit sie Richterroben trugen, der Fahnenflucht, wenn nicht Ignoranz. Oder schlimmer noch: unterstellt ihnen, in »nicht zu übersehender formaler Parallele « zu Praktiken unter dem NS-Regime, sich mit einer »unbegrenzten Auslegung « des Nötigungsparagraphen dem Zeitgeist prostituiert zu haben.

Immerhin bricht der Strafrichter im Unruhestand am Ende dennoch nicht den Stab über seine Gegner. Ein Strafausspruch im Namen des Volkes zu ihren Lasten hätte kaum überrascht. Nein, sich zügelnd sichert er die weiche Flanke des Rechts mit »gegenüber Gesetzen apriorischen Rechtsprinzipien«. So gestärkt, blickt er energisch in die Zukunft und ruft zur Gegenwehr auf, um »eine ganz eindeutig herrschende Auffassung « - exemplarisch hier gemeint: Laepple, das Urteil - vor »dem mit seinen Ideologien je und je anstürmenden Zeitgeist « zu retten.

In der Schlusspassage, auf Kacheln zu brennen, zieht er selbstgenügsam und -gerecht Bilanz: »In der konkreten Situation der Auseinandersetzungen vor dem Amtsgericht Schwäbisch Gmünd um die Strafbarkeit der Teilnahme an den Nachrüstungsblockaden ermöglichte (nur) dieser Ansatz [gemeint: der prinzipiengeleitete des Richters O.- G.F.], die Vielfalt der eingebrachten Argumente in ihrem juristischen Gehalt zu erfassen und kritisch zu sichten, den eigenen Standpunkt zu bestimmen und ihn vor Ort trotz eines bisweilen steifen Gegenwinds zu behaupten. er dürfte damit seine vielleicht exemplarisch bedeutsame Probe bestanden haben."

So kommt Richter O. zu guter Letzt denn doch zu einem Urteil. Freilich zu einem über sich selbst. Und Montesquieu hatte wohl Unrecht: Es geht nichts über den Richter in eigener Sache.

9 So taucht insbesondere der einflussreiche Aufsatz von Brink/Keller zum Gewaltbegriff (KJ I983, I07) nicht auf. Am gescholtenen BVerfG hätte sich Richter O. ein Beispiel für einen weniger selektiven Umgang mit kritischen Stimmen nehmen können (BVerfGE 69, 31 5/344 - Brokdorf - und 73, 206/232 f. Sitzdemonstration). 